\title{
The short nose: Preoperative evaluation and management
}

\author{
Karim Khalil El-Lamie, $M D$ \\ Department of Plastic Surgery, Ain Shams University, Cairo, Egypt.
}

Correspondence:

Assistant professor in plastic surgery department, Ain Shams University.

Address: 3 Beyrouni street, Heliopolis, Cairo, Egypt.

Phone number: 2024183038

E.mail:dr_karimlamie@hotmail.com

\begin{abstract}
Lengthening the short nose is considered the most difficult operation in rhinoplasty. Several techniques have been described ranging from a mere tip graft up to paranasal osteotomies. After preoperative evaluation and measurements, the author suggests a method of lengthening by cartilage grafting using either costal or septal grafts. The type of cartilage grafting depends on the preoperative condition of the nasal septum and the availability of the adequate septal graft. If deemed inappropriate, a costal cartilage graft is harvested and shaped to give the desired effect. By applying the technique in 8 patients, adequate lengthening was achieved in 5 cases. The main obstacle to reach the desired length is the inadequate stretchability of the skin envelope especially after a previous trauma or an operation.

Key words: Short nose, lengthening of the nose, rhinoplasty, caudal septal extension graft, costal cartilage graft, septal graft, tongue in groove technique.
\end{abstract}

\section{Introduction:}

The nasal length is a very important determinant in nasal aesthetics. Most surgeons agree that direct clinical measurement of the nasal length is more useful than anthropometric one. ${ }^{1}$ The normal nose has an appropriate 5:3 ratio between the length and height of the nose. ${ }^{2}$ Lengthening the short nose has always been a dilemma for the plastic surgeon. Several techniques have been suggested using either septal cartilage grafts or costal cartilage grafts to stretch the skin envelope and bring the tip into a more caudal position. ${ }^{3-5}$ Some authors even recommended extensive osteotomies in order to bring the whole nasal complex into a more caudal position. 6,7 However no consensus was made about the basis according to which the surgeon can choose between these methods and if a costal or septal graft are to be used. Also, how each graft can be sculpted and fixed to provide adequate tip support. According to the preoperative examination and CT facial scan, the author suggests a predictable method of lengthening the short nose.

\section{Patients and methods:}

The study was conducted in Ain Shams Hospitals from October 2011 to June 2013, 8 patients were included in the study. The clinical analysis of the cause and anatomy of nasal shortening is essential in planning the corrective surgery. The history of previous trauma or surgical intervention if present is taken with every detail possible. Associated nasal airway complaints are also included in the history taking. The external physical 
examination consisted of a detailed facial aesthetic analysis with emphasis on the nasal length in relation to the midfacial height and nasal projection. Nasal skin laxity and amount of possible skin and mucous membrane stretch is very important as a tight skin and lining is a limitation in some cases for adequate nasal augmentation. Scars in post-traumatic cases were carefully outlined as well as assessment of shape and position of the nasal bone, height of the nasal dorsum, septal support, tip support and width of nasal base. Internal examination included assessment of septal length and any concomitant deviation.

All 8 cases were subjected to clinical measurement of the actual and the desired nasal length. The actual nasal length was measured from the root of the nose at the level of the superior palpebral fold with patient's eye fixed on forward gaze, to the nasal tip. The desired or ideal nasal length is calculated on the basis of midfacial height (MFH) which is the distance from the glabella to the alar base plane. ${ }^{1}$ By multiplying 0.67 the patients measured midfacial height (MFH), the proportioned and ideal nasal length for the face can be calculated i.e. Nasal Length= $0.67 x M F H$. All patients in the study had an actual nasal length shorter by one $\mathrm{cm}$ or more than the ideal nasal length.

Options for correction of the short nose is depending on several factors: the condition of nasal septum (available height and length) which is determined by clinical examination and CT scan, the nasal skin and lining quality and relating it to the desired nasal lengthening and finally concomitant presence of saddle nose deformity. The availability of adequate unmarred septal graft of $2.5 \times 2 \mathrm{~cm}$ is mandatory if correction using septal extension graft is planned otherwise a costal cartilage graft is necessary for the reconstruction. In the latter condition, a $1.5 \mathrm{~mm} \mathrm{~K}$ wire is used to fix the costal cartilage graft to the anterior nasal spine in front of the caudal septum. In both types of grafts, the medial and middle crura are then sutured in the midline overlapping the graft leading to distalization of the nose.

\section{Surgical technique:}

Using the open approach, the nasal skin is dissected completely to the level of the nasofacial crease horizontally and nasofrontal angle vertically. The periosteum is elevated from the dorsum of nasal bones, lateral and/ or medial osteotomies are performed when indicated. The septum is dissected using extra-mucosal tunneling technique and the septal graft is harvested if judged possible leaving an inverted $\mathrm{L}$ strut of $1 \mathrm{~cm}$ in width. Otherwise, a $5 \mathrm{~cm}$ costal cartilage graft is harvested from the $5^{\text {th }}$ rib. To correct the foreshortened nose deformity using septal extension graft, the latter is shaped so that it has a longer superior margin, usually by $5 \mathrm{~mm}$, than its base. The graft cephalic border is beveled and overlapped by around $7 \mathrm{~mm}$ to the caudal margin of the caudal septum using 2 polypropylene 4-0 mattress sutures. For further support the inferior edge of the graft is sutured to the anterior nasal spine periosteum.Utmost attention is paid to ensure that the caudal margin of the graft sits in the midline Figure(1). In case of costal cartilage graft a $25 \mathrm{~mm}$ length $\mathrm{x} 10 \mathrm{~mm}$ width and $6 \mathrm{~mm}$ thick cylinder is carved from the graft and penetrated by a $\mathrm{K}$ wire before being fixed to the anterior nasal spine. The angulation at which the costal graft is fixed determines the amount of tip distalization. In case of concomitant depressed nasal bridge another graft is sculpted from the costal cartilage and used in onlay fashion after being penetrated by a $\mathrm{K}$ wire to prevent warpage.

The skin is then redraped and columellar incision is temporarily sutured to check adequate skin laxity. If skin coverage is deemed too tight, the septal extension graft is trimmed sequentially until adequate closure is achieved. For the costal cartilage graft, the $\mathrm{K}$ wire is bent slightly cephalically to allow approximation of the columellar incision. Once skin closure is ensured, the columellar suture is removed and the medial and middle crura are advanced with the attached nasal lining and sutured tongue in groove fashion to the caudal margin of the graft. If the lining is deficient, the mucosa is further dissected from the bony septum. If still the lining is tight and hindering the caudal movement of the 
alar cartilage, with a double hook on the tip cartilage, a knife is used to release the dorsal aspects of the mucoperichondreal flaps. Up to three staggered cuts $1 \mathrm{~cm}$ in length and spaced by $1.5 \mathrm{~cm}$ are made. By staggering the releasing cuts, the cartilaginous septum will always have a mucosal lining on at least one side.

The lower lateral cartilages are now sutured to the caudal end of either septal or costal cartilage grafts in a "tongue in groove" fashion and further tip refining sutures are used for a more pleasing result. In 5 of the 8 patients shield grafts harvested from the ear concha were also added to the tip area for a better tip projection and definition Figure(2).

\section{Results:}

8 patients (6 males and 2 females) were included in the study with 5 receiving septal extension grafts and 3 costal cartilage grafts for lengthening a foreshortened nose Table(1). 6 patients were of posttraumatic etiology, one patient had a previous rhinoplasty with overzealous septal resection while 1 patient had no history of any previous trauma. The maximum lengthening achieved was $10 \mathrm{~mm}$ and the least was $6 \mathrm{~mm}$ Figure(3). 5 patients reached the ideal nasal length while 3 patients were short from this goal. The main if not the only the cause for this inadequate lengthening was the tightness of the skin envelope. All 3 patients had a history of relatively recent trauma of less than 2 years duration Figure(4). Two of the three patients receiving costal cartilage graft had concomitant saddle nose deformity and received an onlay dorsal graft. All patients were followed for 6 months at least and showed no postoperative complication such as infection, abnormal bleeding, nasal airflow obstruction, grafts displacement or extrusion of $\mathrm{K}$ wires.

\section{Discussion:}

Several causes of short nose are known: congenital, trauma, cocaine addiction, and iatrogenic. ${ }^{8}$ Lengthening the short nose is arguably the most difficult operation in aesthetic rhinoplasty. The apparently short nose is characterized by: (l) an obtuse columellar-labial angle; (2) an overprojecting tip; and (3) a shallow slope (nasofacial angle) or the dorsum. ${ }^{2}$ The normal nose has an approximately 5:3 ratio between the length and height of the nose. However since the tip projection is usually altered in the short nose, Byrd et al introduced the midfacial height as way to evaluate the adequate nasal length. Ideally the nasal length is 0.67 that of the midfacial height. ${ }^{1}$ In the past using the closed technique, the lengthening was very limited. Without soft tissue release, lengthening with cartilage grafts was compromised by the stretchability of the tissues. Moreover much of the gained length was lost in the postoperative period because the soft tissues (and the contraction process) simply pulled the nose back towards its original length sometimes with a resultant deviation. ${ }^{9}$ The open rhinoplasty approach allowed for complete intraoperative evaluation of the condition beside complete and adequate release of both the mucoperichondrial lining as well as skin envelope. ${ }^{10}$ Many authors suggested different methods of lengthening of the nose in mild condition (less than $0.3 \mathrm{~cm}$ ) such as: release of the alar cartilages from the upper lateral cartilages, composite graft, batten graft, and onlay tip grafts. $2,11,12$ A septal extension graft or costal cartilage graft was advised for cases between 3-5 mm lengthening. ${ }^{3-5,13}$ For more lengthening some authors suggested paranasal osteotomies to bring the entire nasal complex forward.6,7 The rationale behind this decision is that the lining and skin coverage would not allow for such augmentation. In this study, all patients included required nasal lengthening more than $5 \mathrm{~mm}$ and all were managed by either septal extension graft or costal cartilage graft. The decision between both types of grafts were based on the condition of septum primarily which was evaluated both clinically and radiologically by CT scan. Many authors suggested that for better support a costal cartilage graft is favored. However according to the results, both grafts offered equal and stable nasal tip lengthening provided that the septal extension graft is adequately harvested and fixed to the caudal septum. The nasal 


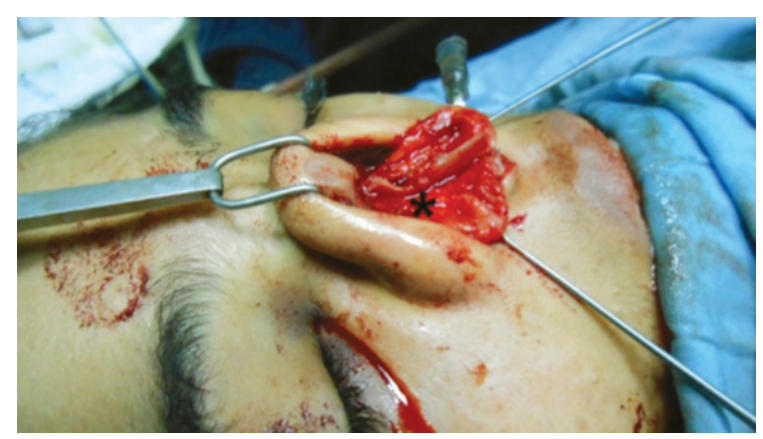

Figure (1): Intraoperative view showing the septal extension graft after the fixation the actual caudal septum marked by the star. The lower lateral cartilages with the lining mucosa are stretched by the two hooks and fixed to the end of the graft by the syringe needle.
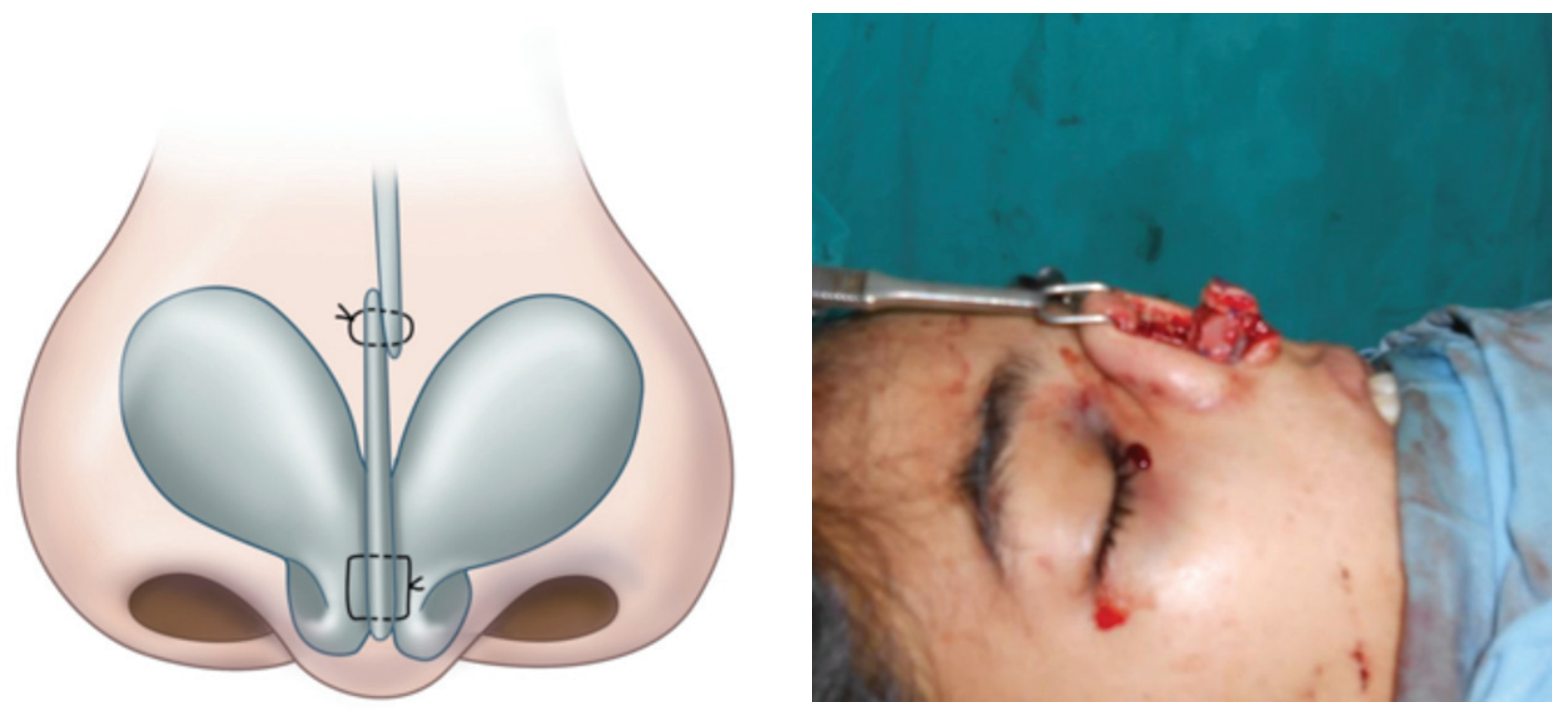

Figure (2): (Left) The septal extension graft is overlapped on both sides and sutured in "tongue in groove" fashion to the medial and middle crura of the lower lateral cartilages. (Right) Further tip definition and augmentation can be achieved by onlay shield cartilage graft taken from the ear concha.
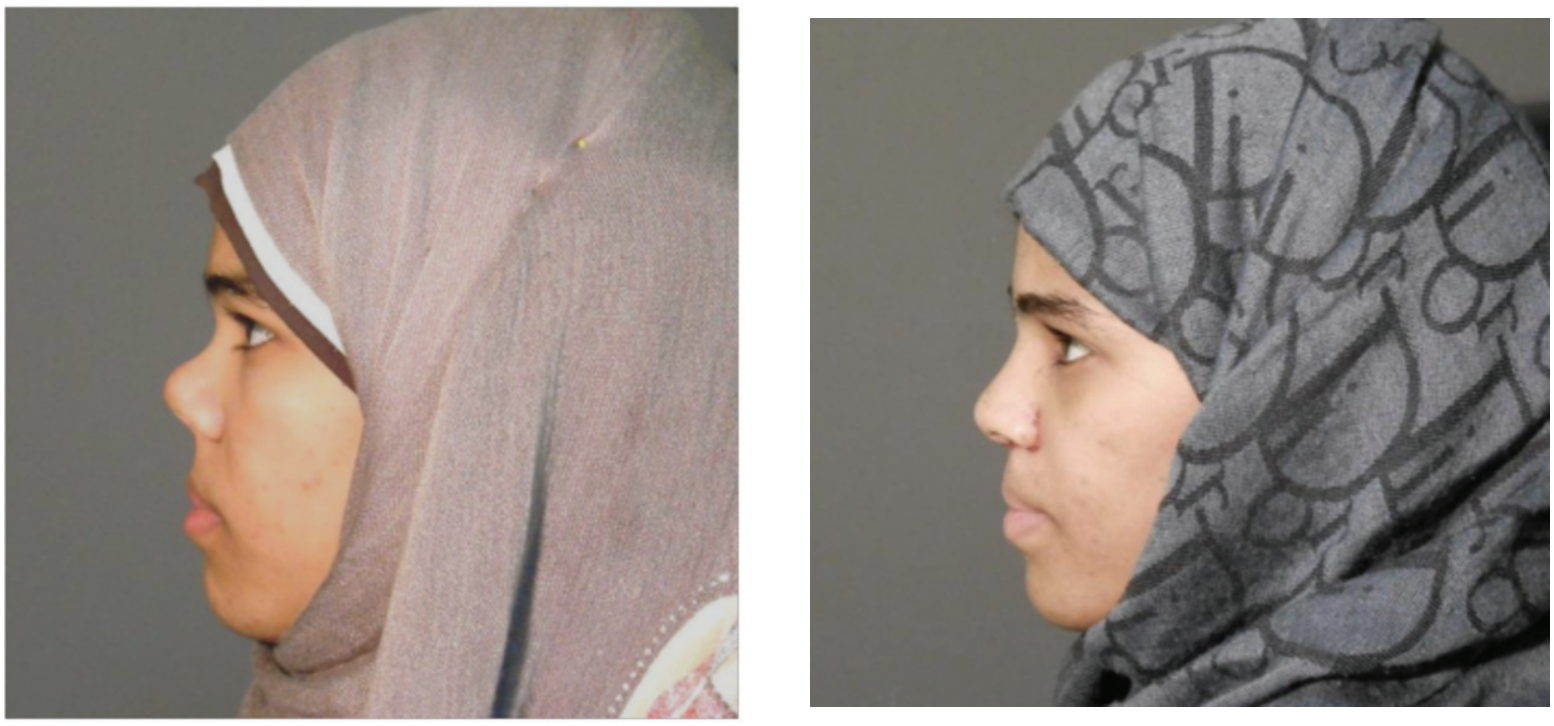

Figure (3): (Left) Preoperative side view of an 18 year old female patient presenting with a post traumatic short nose after a fall on her face at the age of 4 years. (Right) Postoperative view after using a septal cartilage extension graft and tip conchal cartilage graft. The nasal lengthening achieved was $10 \mathrm{~mm}$. 

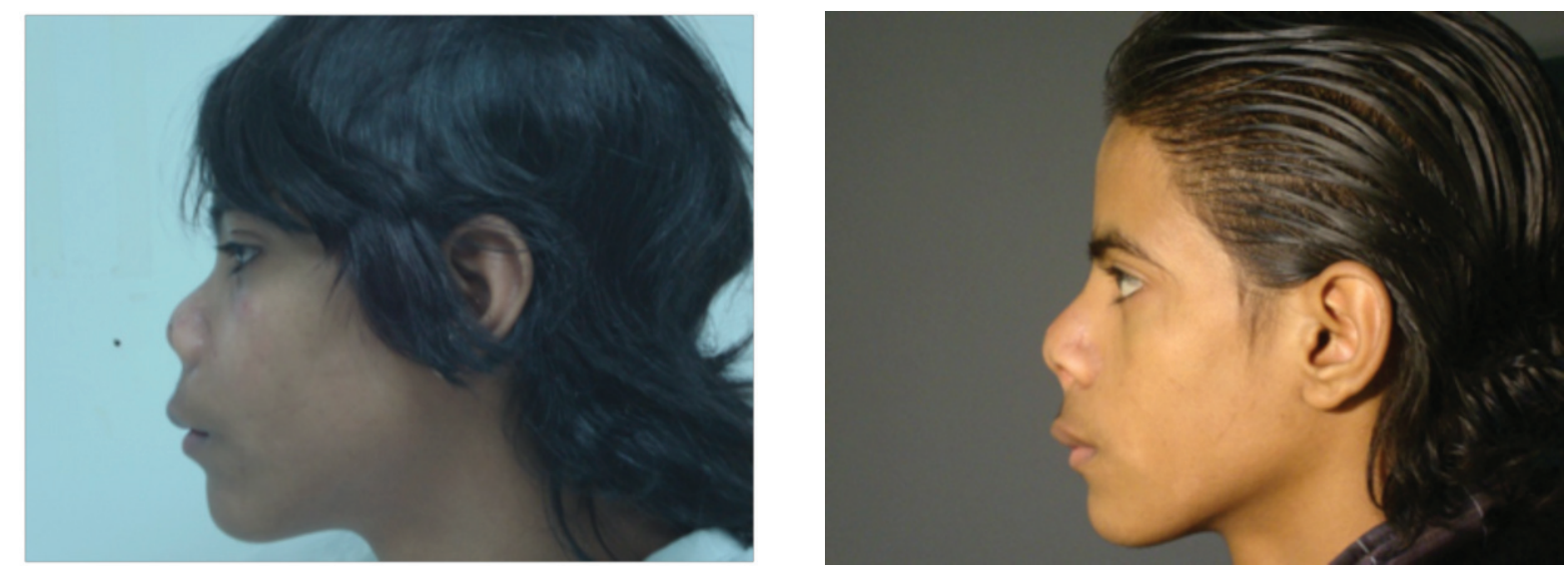

Figure (4): (Left) Preoperative view of a 17 year old male with history of severe trauma to the nose of 15 months duration and wound repair elsewhere. (Right) Postoperative view after costal cartilage grafting for both nasal lengthening and for dorsal augmentation. The achieved lengthening was $6 \mathrm{~mm}, 3 \mathrm{~mm}$ shorter than the desired goal.

Table (1): Data of patients.

\begin{tabular}{|c|l|c|l|c|c|c|}
\hline $\begin{array}{c}\text { Patient } \\
\text { number }\end{array}$ & Sex & Age & Etiology & $\begin{array}{c}\text { Cartilage } \\
\text { used }\end{array}$ & $\begin{array}{c}\text { Lengthening } \\
\text { required }\end{array}$ & $\begin{array}{c}\text { Lengthening } \\
\text { achieved }\end{array}$ \\
\hline 1 & Male & 17 & Trauma & Costal & $6 \mathrm{~mm}$ & $6 \mathrm{~mm}$ \\
\hline 2 & Male & 18 & Trauma & Septal & $7 \mathrm{~mm}$ & $7 \mathrm{~mm}$ \\
\hline 3 & Male & 20 & Trauma & Septal & $10 \mathrm{~mm}$ & $7 \mathrm{~mm}$ \\
\hline 4 & Female & 17 & Iatrogenic & Costal & $8 \mathrm{~mm}$ & $6 \mathrm{~mm}$ \\
\hline 5 & Male & 16 & Congenital & Septal & $8 \mathrm{~mm}$ & $6 \mathrm{~mm}$ \\
\hline 6 & Male & 17 & Trauma & Costal & $9 \mathrm{~mm}$ & $6 \mathrm{~mm}$ \\
\hline 7 & Male & 23 & Trauma & Septal & $7 \mathrm{~mm}$ & $7 \mathrm{~mm}$ \\
\hline 8 & Female & 18 & Trauma & Septal & $10 \mathrm{~mm}$ & $10 \mathrm{~mm}$ \\
\hline
\end{tabular}

lining was mainly a problem in the iatrogenic case but not in the other post-traumatic patients. Adequate release of the lining with the staggered cuts allowed for adequate stretch in all post-traumatic cases without any postoperative relative drawbacks. Worst outcome was mainly seen in those who had relatively recent nasal trauma (less than 2 years) and those who had undergone an inadequate rhinoplasty with excessive septal shortening. The main problem was in the skin coverage which was found stiff and incompliant for 3 patients, however still more than $5 \mathrm{~mm}$ lengthening was still achievable. One of the patients followed for more than a year was found to have the skin becoming more resilient after that edema had subsided. Another minor procedure of augmentation can be contemplated later on rather than jumping to a major operation like the paranasal osteotomies from the start.

\section{Conclusion:}

Management of the short nose requires both very good preoperative planning as well as mastering rhinoplasty skills. Extensive dissection and release of all nasal components, ability to harvest an adequate septal or costal cartilage graft, and mastering tip definition techniques are all prerequisites for attempting such surgery. From the point of view of strength and tip support, there is no difference 
between the septal and costal cartilage graft as long as the septal graft is adequately harvested, shaped and sutured to the caudal septum. The author suggests his technique of both septal and costal cartilage grafting. The main hinder of adequate lengthening was found primarily in the skin envelope especially in those iatrogenic or recent posttraumatic patients. Further follow-up of these patients with inadequate lengthening suggests that another minor procedure can be planned later on.

\section{References:}

1- Byrd SH, Burt JD, Yazdani A, El-Musa KA: Lengthening the short nose. In: Dallas rhinoplasty nasal surgery. Gunter JP, Rohrich RJ, Adams, WP Jr (Editors); St Louis Misouri: Masters (Publisher); 2nd edn.; Vol. 3; 2007; p.1049-1070.

2- Gruber RP: Lengthening the short nose. Plast Reconstr Surg 1993; 91(7): 1252-1258.

3- Toriumi DM, Hecht D: Skeletal modification in rhinoplasty. Facial Plast Surg Clin North Am 2000; 4: 413-431.

4- Hubbard TJ: Exploiting the septum for maximal tip control. Ann Plast Surg 2000; 44(2): 173-180.

5- Burgett GC: Costal cartilage for nasal reconstruction through a small incision. Clin Plast Surg 1993; 7: 129-136.
6- Converse JM: Surgical elongation of the traumatically foreshortened nose. The perinasal osteotomy. Plast Reconstr Surg 1971; 47(6): 539-546.

7- Wolfe SA: Lengthening the nose: A lesson from craniofacial surgery applied to posttraumatic and congenital deformities. Plast Reconstr Surg 1994; 94(1): 78-87.

8- Lee Y, Kim J, Lee E: Lengthening of the postoperative short nose: Combined use of a gull-wing concha composite graft and a rib costochondral dorsal onlay graft. Plast Reconstr Surg 2000 May; 105(6): 2190-2199.

9- Rohrich R: Nasal lengthening with extended spreader grafts. Presented at the American Society for Aesthetic. Plastic Surgery Meeting, San Francisco, 1995.

10-Whitaker EG, Johnson CM Jr: The evolution of open structure rhinoplasty. Arch Facial Plast Surg 2003; 5(4): 291-300.

11-Gunter JP, Rohrich RJ: Lengthening the aesthetically short nose. Plast Reconstr Surg 1989; 83(5): 793-800.

12-Dingman RO, Walter C: Use of composite ear grafts in correction of the short nose. Plast Reconstr Surg 1969; 43 (2): 117-218.

13-Chang YL Correction of difficult short nose by modified caudal septal advancement in Asian patients. Aesthet Surg J 2010; 30(2): 166-175. 